Im „Journal Club“ werden Originalarbeiten aus der internationalen Fachliteratur referiert und kommentiert.

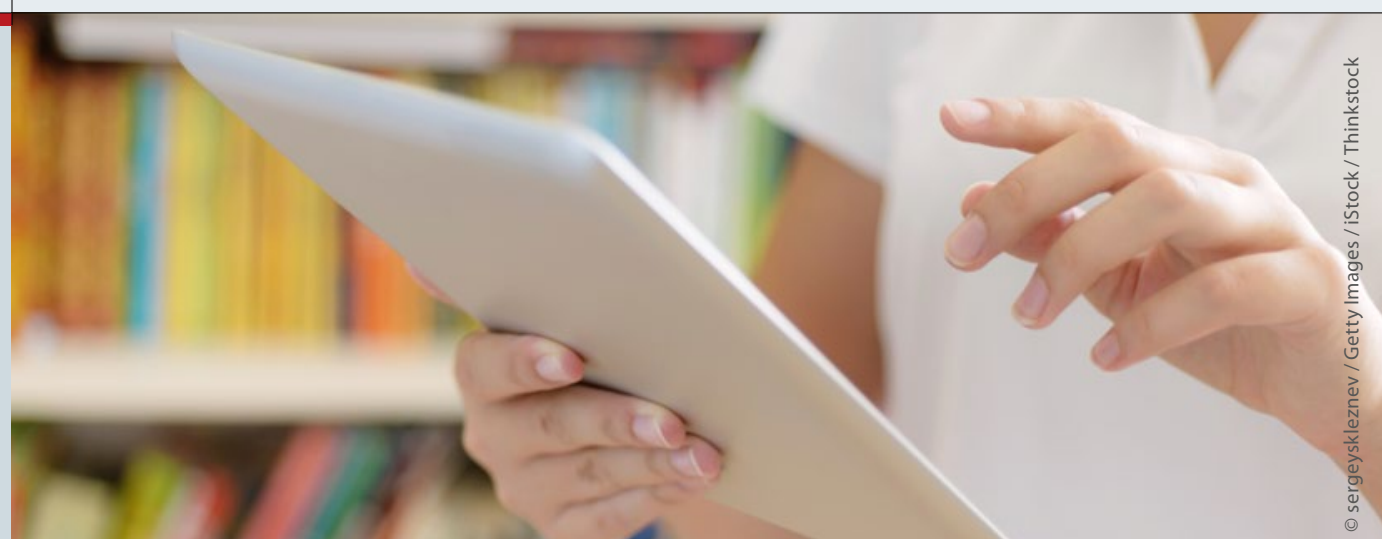

\section{Entscheidungskriterien beim leichten Schädel-Hirn-Trauma}

\begin{abstract}
Wird ein Kind wegen eines leichten Schädel-Hirn-Traumas notfallmäßig vorgestellt, muss geklärt werden, ob der Patient wieder nach Hause darf oder ob eine stationäre Überwachung nötig ist. Eine Münchner Gruppe von Kinderchirurgen hat jetzt untersucht, wie diese Entscheidung getroffen werden kann.
\end{abstract}

D as leichte Schädel-Hirn-Trauma zählt zu den häufigsten Verletzungen von Kindern in der Notfallversorgung. Nicht immer lassen die ersten Symptome genaue Rückschlüsse auf den Schweregrad der Verletzungen zu. Deshalb stellt sich auch bei einem leicht erscheinenden Krankheitsbild zunächst die Frage, ob das Kind nach der Untersuchung mit den über mögliche Symptome aufgeklärten Eltern wieder nach Hause geschickt werden kann oder ob es stationär aufgenommen werden muss.

In einer retrospektiven Analyse haben Danielle Wendling-Keim und Kollegen vom Dr. von Haunerschen Kinderspital der Universität München die Zuverlässigkeit der Risikoabschätzung bei Schädel-Hirn-Trauma in Anlehnung an die PECARN(Pediatric Emergency Care Applied Research Network)-Regeln untersucht. Ziele waren die sichere Erkennung schwerwiegender Gehirnverletzungen, eine niedrige Komplikationsrate, eine hohe Patientenzufriedenheit sowie das möglichst seltene Auftreten eines postkontusionellen Syndroms.

\section{Sturz vom Wickeltisch, Fahrrad oder Sofa}

Insgesamt 267 Familienangehörige von Kindern im durchschnittlichen Alter von 4,1 Jahren mit einem Schädel-HirnTrauma 1. Grades (Glasgow Coma Scale, GCS-Score: 13-15 Punkte) hatten nachträglich einen Fragebogen beantwortet.
140 der Kinder waren ambulant untersucht und unter Beobachtung der instruierten Eltern wieder nach Hause geschickt, 127 in der Klinik überwacht worden. Die meisten Patienten waren, je nach Alter, vom Wickeltisch oder Sofa gefallen, vom Fahrrad oder Roller gestürzt oder mit irgendeinem Gegenstand kollidiert.

Die Kinder, die ambulant versorgt wurden, fielen symptomatisch lediglich durch Kopfschmerzen auf. Bei 9\% dieser Patienten wurde ein bildgebendes Verfahren durchgeführt (bis 18 Monate Sonografie durch die Fontanelle, danach CT oder MRT), doch in keinem Fall zeigte sich eine Auffälligkeit. Die Befragung ergab, dass fast drei Viertel der Kinder und Jugendlichen nach der ambulanten Behandlung keine weitere ärztliche Hilfe benötigt hatten.

Bei den stationär behandelten Kindern wurden hauptsächlich folgende Symptome angegeben: Übelkeit und Erbrechen (55\%), Bewusstseinsstörungen, Amnesie (35\%), Kopfschmerzen (9\%), Schwindel (5\%). Bei 7,4\% von ihnen war Bewusstlosigkeit von maximal einer Minute aufgetreten. 37\% dieser Gruppe waren einer Bildgebungsdiagnostik unterzogen worden, aber bei nur drei Patienten ergab sich ein pathologischer Befund. Dabei handelzwei Fällen mit intrakraniellen Blutungen einhergingen. 94\% der stationär versorgten Kinder wurden nach ein bis zwei te es sich um Schädelfrakturen, die in
Tagen entlassen. Die meisten (65\%) mussten sich danach keiner weiteren ärztlichen Behandlung unterziehen.

Nur zehn der ambulanten und stationären Patienten entwickelten ein postkontusionelles Syndrom. Sowohl nach ambulanter als auch nach stationärer Versorgung war die Patientenzufriedenheit sehr hoch.

\section{Unter zwei Jahren großzügig stationär aufnehmen}

Den Ergebnissen ihrer Studie zufolge sehen Wendling-Keim und Kollegen einen Algorithmus in Anlehnung an die PECARN-Regeln als sichere Grundlage bei der Entscheidung für oder gegen die Notwendigkeit einer stationären $\mathrm{Be}$ handlung nach Schädel-Hirn-Trauma. Alle Patienten unter sechs Monaten mit einem Kopftrauma sollten grundsätzlich stationär aufgenommen werden. Bei älteren Kindern hänge diese Entscheidung davon ab, ob Symptome wie Übelkeit und Erbrechen, fokal-neurologische Defizite, Bewusstseinsstörungen, eine Schädelfraktur oder Kopfhauthämatome vorlägen, so Wendling-Keim und Kollegen. Kinder unter zwei Jahren sollten aber grundsätzlich großzügig eingewiesen werden, da sich diese möglicherweise noch nicht so gut äußern könnten und in diesem Alter nach einem Kopftrauma ein erhöhtes Risiko intrakranieller Verletzungen bestehe. Da man allgemein davon ausgeht, dass eine Hirnblutung innerhalb von 24-48 Stunden entsteht, halten die Kinderchirurgen eine Beobachtungszeit über diese Zeitspanne für angebracht.

Wendling-Keim D S et al. Ambulatory or inpatient management of mild TBI in children: a postconcussion analysis. Pediatr Surg Int 2016; online 17. November 2016; DOI 10.1007/s00383016-4021-z 